\title{
Antenna Diversity for a Narrow-Band Successive-Cancellation Multiuser Detector
}

\author{
Gerard J. M. Janssen, Member, IEEE, and S. Ben Slimane, Member, IEEE
}

\begin{abstract}
The performance of a successive-cancellation multiuser detector (SC-MUD) for narrow-band signals operating in the Rayleigh-fading channel is shown to deteriorate severely unless very large power margins at the receiver are maintained. To mitigate this deterioration, antenna diversity is applied and adapted to the SC-MUD. In each detection stage, the best signal for detection and the corresponding combining weights are determined jointly. Two criteria for selecting the best signal based on maximizing the signal-to-noise-plus-interference ratio and maximizing the minimum distance-to-noise ratio, respectively, are proposed and evaluated. For signal combining, zero forcing and minimum mean square error (mmse) combining are considered. The obtained results show that the SC-MUD with mmse combining performs close to maximum likelihood joint detection while keeping a much lower computational complexity when the number of users is less than the number of receive antennas. The effect of channel-estimation errors is investigated for orthogonal training sequences and shown to result in a minor degradation compared to perfect estimation.
\end{abstract}

Index Terms-Antenna diversity, channel estimation, narrowband multiuser detection (MUD), successive cancellation, symbol error probability (SEP).

\section{INTRODUCTION}

$\mathbf{M}$ ULTIUSER DETECTION (MUD) research has been mainly related to code-division multiple access (CDMA) [1] for reducing multiple-access interference in order to increase spectral efficiency. Recently, MUD receivers have been proposed for narrow-band $M$-ary phase-shift keying ( $M$-PSK) modulated cochannel signals based on successive cancellation [2]-[4] and maximum-likelihood (ML) detection [5]-[7]. By narrowband we refer to the fact that, unlike in CDMA, no bandwidth-expanding signature code is applied to separate the user signals.

In the successive-cancellation MUD (SC-MUD), the largest signal is detected and estimated in the first detector; this estimate is used to cancel the detected signal from the total input signal. Subsequently, the next largest signal is detected, estimated, and canceled, and so on. A detection error results in error propagation to all signals that are still to be detected. The structure of the SC-MUD is shown in Fig. 1. For a low symbol error

Manuscript received January 28, 2003; revised August 27, 2003, January 21, 2004, and March 22, 2004.

G. J. M. Janssen is with the Wireless and Mobile Communications Group, Department of Electrical Engineering, Mathematics, and Computer Science (EEMCS), Delft University of Technology, Delft 2628 CD, The Netherlands (e-mail: g.janssen@ewi.tudelft.nl).

S. Ben Slimane is with the Radio Communication Systems Group, Department of Signals, Sensors and Systems, Royal Institute of Technology, Stockholm SE-164 40, Sweden (e-mail: slimane@ radio.kth.se).

Digital Object Identifier 10.1109/TVT.2004.832372 probability (SEP), sufficient power differences between the signals at the receiver input are required. In nonfading or slowly fading channels, power control can be applied to maintain these power differences. In [8], application of an SC-MUD in a cellular system with centralized power control is shown to result in a substantial increase of the system capacity. In the case of fast multipath fading, however, the required power differences cannot be maintained, which results in a poor performance for all user signals. Increasing the power differences between the signals for maintaining a desired SEP requires very large power margins, as shown in Section III.

To mitigate this problem, antenna diversity can be applied at the receiver. Optimum detection performance for narrow-band signals using multiple antennas is obtained by ML joint detection (ML-JD), as proposed in [7]. The detected compound symbol of $K$ users is the one that has minimum Euclidean distance to the received compound symbol. This technique performs very well in the Rayleigh-fading channel; however, its complexity is $O\left(L M^{K}\right)$ where $L$ is the number of antennas and $M$ is the modulation level, i.e., the complexity increases exponentially with the number of users $K$.

In this paper, we apply and adapt antenna diversity to the SC-MUD in the uplink. Since the users transmit independent data streams from different locations over uncorrelated channels, this scenario can be seen as a special case of the V-BLAST scheme [9], [10]. In each detection stage, the optimum signal for detection is determined (i.e., the signal that results in the lowest SEP), as well as the optimum combining weights. For combining we compare zero-forcing (ZF) and minimum mean square error (mmse) combining. The complexity of this receiver is $O(L M K)$ since in each stage only the symbol of the intended user is detected, i.e., the complexity increases linearly with the number of users $K$. Simulations show that the performance of the SC-MUD using mmse combining approaches the performance of the ML-JD for $K<L$. We further analyze the effect of channel-estimation errors on the SEP when using short orthogonal training sequences. It is shown that channel-estimation errors cause a limited performance degradation compared to perfect estimation.

The system model for the SC-MUD with multiple receive antennas, is given in Section II. In Section III, the SEP model is presented for the SC-MUD with antenna diversity, taking into account error propagation and channel-estimation errors. In Section IV, we propose two signal-selection criteria and combine these with ZF and mmse combining. Performance results obtained from simulations are presented in Section $\mathrm{V}$ and a conclusion is drawn in Section VI. 


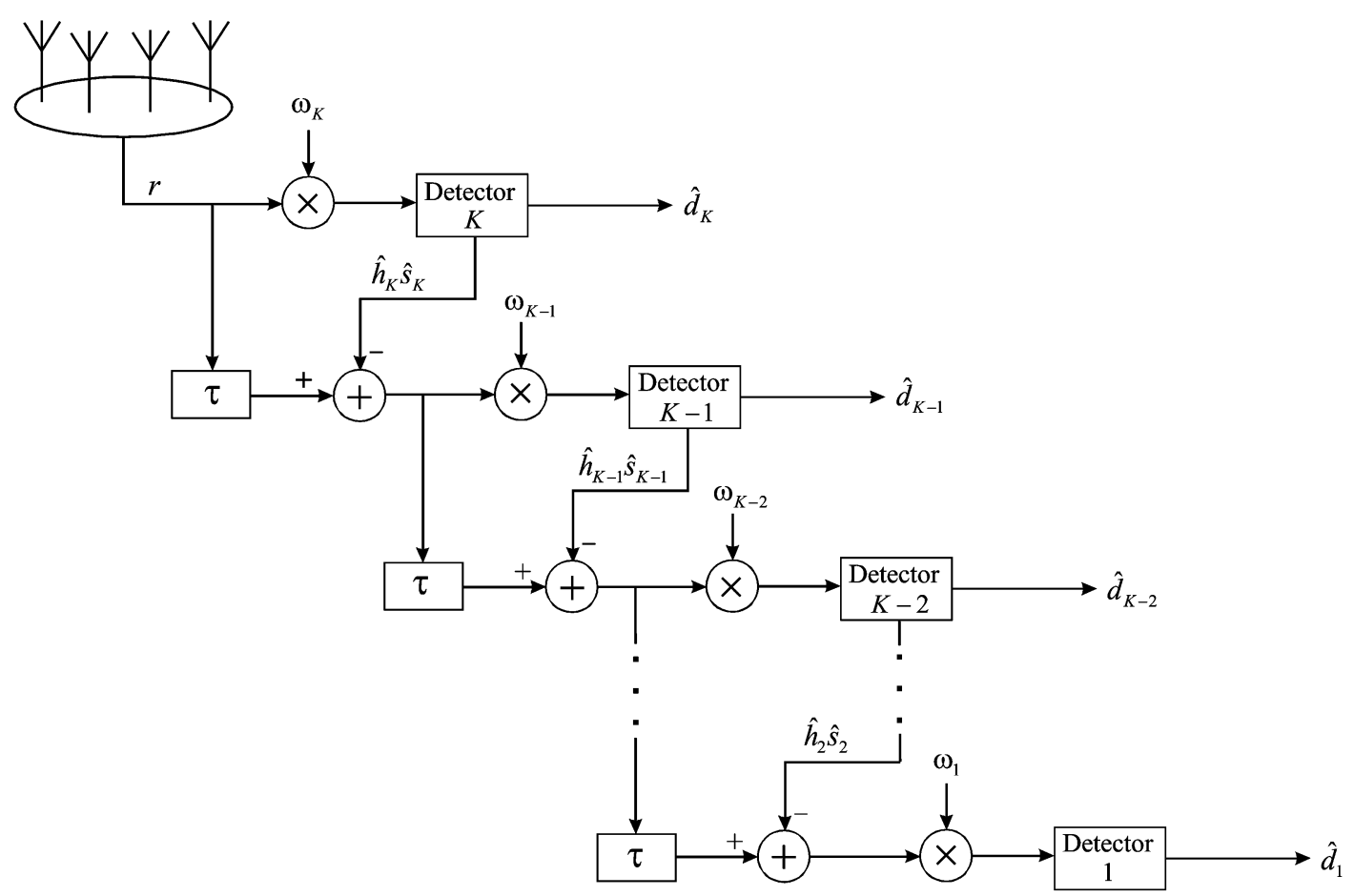

Fig. 1. Block diagram of the narrow-band SC-MUD with antenna diversity.

\section{SySTEM MODEL}

We consider a symbol-synchronous multiuser system with $K$ active noncoherent users in the uplink, all using the same carrier frequency and $M$-PSK modulation. For such a structure, the bandwidth efficiency $\rho=K \log _{2}(M)[\mathrm{bits} / \mathrm{s} / \mathrm{Hz}]$ increases linearly with the number of users per channel. The equivalent lowpass transmitted signal of user $k$ during the $n$th symbol interval $T_{s}$ is given by

$$
s_{k}(t)=A_{k} e^{j a_{k}(n) \frac{2 \pi}{M}}, \quad n T_{s} \leq t<(n+1) T_{s}
$$

where $A_{k}=\sqrt{E_{k} / T_{s}}$ is the amplitude, $E_{k}$ is the transmitted symbol energy, and $a_{k}(n)=0,1, \ldots, M-1$ with equal probability of occurrence. The signal is transmitted in bursts, part of which consists of a training sequence for channel estimation.

The channel is modeled as a flat-fading frequency-nonselective channel with complex amplitude gain $h=|h| e^{j \theta}$. The amplitude $|h|$ is Rayleigh distributed and the phase $\theta$ is uniformly distributed over $[0,2 \pi)$. In the following, we neglect path loss and shadowing effects and assume normalized channel fading coefficients, i.e., $\overline{|h|^{2}}=1$; thus, the expected received symbol energy for user $k$ is equal to the transmitted symbol energy. We further assume a slowly varying fading channel, which can be considered constant during the transmission of a burst.

The receiver (e.g., a base station) consists of an $L$-element antenna array of arbitrary structure. The array elements are sufficiently spaced apart so that the Rayleigh fading can be considered mutually independent. Thus, each branch receives independent replicas of all user signals. The complex channel gain between array element $l$ and user $k$ is given by $h_{l, k}$. Now the equivalent lowpass representation of the received signal at any of the array elements at the receiver is written as

$$
r_{l}(t)=\sum_{k=1}^{K} h_{l, k} s_{k}(t)+n_{l}(t), \quad l=1,2, \ldots, L
$$

where $n_{l}(t)$ is the additive white Gaussian noise (AWGN) process at antenna element $l$ having zero mean and power spectral density $N_{0}$. Passed through a matched-filter demodulator, the signal of antenna element $l$ during the $n$th symbol interval is given by

$$
r_{l}(n)=\sum_{k=1}^{K} h_{l, k} s_{k}(n)+n_{l}(n), \quad l=1,2, \ldots, L
$$

where $n_{l}(n)$ now is a complex Gaussian random variable with zero mean and variance $N_{0}$. For ease of notation, we omit the symbol index $n$ in the following.

Let us define the $L \times K$ channel matrix $H$ as

$$
\boldsymbol{H}=\left[\begin{array}{cccc}
h_{1,1} & h_{1,2} & \cdots & h_{1, K} \\
h_{2,1} & h_{2,2} & \cdots & h_{2, K} \\
\vdots & \vdots & \vdots & \vdots \\
h_{L, 1} & h_{L, 2} & \cdots & h_{L, K}
\end{array}\right]
$$

the vector $s=\left[s_{1}, s_{2}, \ldots, s_{K}\right]^{T}$, which contains the transmitted signals, and the noise vector $\boldsymbol{n}=\left[n_{1}, n_{2}, \ldots, n_{L}\right]^{T}$, which contains independent and identically distributed (i.i.d.) Gaussian noise samples, where $(\cdot)^{T}$ denotes the matrix transpose. Now the vector $\boldsymbol{r}=\left[r_{1}, r_{2}, \ldots, r_{L}\right]^{T}$ of the signals received at the antenna elements can be written as

$$
\boldsymbol{r}=\boldsymbol{H s}+\boldsymbol{n}
$$

The received vector above is used at the receiver to separate the symbols of the different users and to make a decision. A straightforward method to achieve this goal is by using ML-JD, which allows optimum detection for the different user symbols, but has a complexity that increases exponentially with the number of users. As an alternative solution, successive cancellation can be used instead. The redundancy provided by the dif- 
ferent antennas together with some power margins between the different signals will make such a detection procedure possible and reliable. Both ML-JD and successive cancellation require knowledge of the channel-state information at the receiver.

\section{A. Channel Estimation}

The channel estimates are usually not perfect due to the presence of noise and other cochannel signals and will not allow perfect signal cancellation even in the absence of symbol errors. Here, we extend the channel-estimation model presented in [3] to the application of antenna diversity.

We assume that the user signal is transmitted in short bursts with $N$ symbols per burst, part of which is a training sequence of $J(J<N)$ consecutive symbols. We further assume that the training sequences, denoted $\left\{w_{k}(t)\right\}$, of the different users are orthogonal with

$$
\int_{0}^{J T_{s}} w_{i}(t) w_{k}^{*}(t) d t=\left\{\begin{array}{ll}
J, & i=k \\
0, & i \neq k
\end{array} .\right.
$$

Thus, for symbol and burst-synchronous multiuser systems, the inaccuracy in parameter estimation is caused by additive noise only. Note that nonorthogonal training sequences and/or nonperfect synchronization between user signals will introduce additional estimation errors. The equivalent lowpass of the received signal at array element $l$, containing the training sequences of the active signals, during a particular burst can be written as

$$
r_{l}(t)=\sum_{k=1}^{K} A_{k} h_{l, k} w_{k}(t)+n_{l}(t), \quad 0 \leq t \leq J T_{s}
$$

where $w_{k}(t)$ is the transmitted training sequence of signal $k, h_{l, k}$ is the complex channel response assumed constant during a burst, and $n_{l}(t)$ is a zero-mean complex Gaussian noise process at array element $l$ with spectral density $N_{0}$.

The complex channel response $h_{l, k}$ is estimated by using the $J$ training sequence symbols as

$$
\hat{h}_{l, k}=\frac{1}{J \cdot A_{k}} \int_{0}^{J T_{s}} r_{l}(t) w_{k}^{*}(t) d t=h_{l, k}+\frac{\tilde{n}_{l, k}}{A_{k}}
$$

where $\hat{h}_{l, k}$ is the estimate of $h_{l, k}$. By averaging over the $J$ symbols of the training sequence, the effect of noise is reduced by a factor of $J$. In that, the noise component $\tilde{n}_{l, k}$ is a complex Gaussian random variable with zero mean and variance $N_{0} / J$. In practice, we will usually estimate the parameters of the received signal $k$ at element $l$, given by $A_{k} h_{l, k}$.

For evaluation of the effect of channel-estimation errors on the performance of the SC-MUD, we generate the channel estimate $\hat{h}_{l, k}$ as

$$
\hat{h}_{l, k}=h_{l, k}+\varepsilon_{l, k}
$$

where $\varepsilon_{l, k}$ is a complex zero-mean Gaussian variable given by $\varepsilon_{l, k}=\tilde{n}_{l, k} / A_{k}$ with variance $\sigma_{\varepsilon_{l, k}}^{2}=N_{0} /\left(J A_{k}^{2}\right)$. Note that the estimation error variance is inversely proportional to the transmitted signal power and the length of the training sequence.
Since the training sequences are orthogonal, the terms $\varepsilon_{l, k}$ are independent Gaussian random variables [11], [12] at every antenna element $l$.

\section{SuCCESSIVE-CANCELLATION MULTIUSER DETECTION WiTH ANTENNA COMBINING}

The narrow-band SC-MUD for multiple $M$-PSK modulated signals, which was originally proposed in [2], is based on successive signal detection, estimation, and cancellation. The structure of the receiver with antenna combining is shown in Fig. 1. After combining, a selected signal $k$ is detected and estimated in detector $K$. This estimate is then used to cancel signal $k$ in each of the antenna branches, which can be done because its complex signal amplitude $A_{k} \hat{h}_{l, k}$ is known at each branch $l$. In the following stage $K-1$, a new weight vector is determined, optimized for one of the remaining signals that, after combining, is subsequently detected, estimated, subtracted, and so on. The stage number refers to the number of signals left for detection in that stage. This principle is known as "onion peeling" [13]. In practice, the optimum signal to be detected is not known in advance because of the fading, but has to be selected in each detection stage. Strategies for the detection order are discussed in Section IV.

\section{A. Successive Cancellation}

Let us consider stage $p$ of the SC-MUD, when $K-p$ signals have already been detected and canceled. In this stage, the $p$ signals that still need to be detected are indicated as $s_{p, i}, i=$ $1, \ldots, p$. The received vector at this stage can be written as

$$
\boldsymbol{r}_{p}=\boldsymbol{H}_{p} \boldsymbol{s}_{p}+\boldsymbol{H}_{K-p} \boldsymbol{s}_{K-p}-\hat{\boldsymbol{H}}_{K-p} \hat{\boldsymbol{s}}_{K-p}+\boldsymbol{n}
$$

where the vector $\boldsymbol{s}_{p}=\left[s_{p, 1}, s_{p, 2}, \ldots, s_{p, p}\right]^{T}$ denotes the set of remaining signals still to be detected, the vector $\boldsymbol{s}_{K-p}=$ $\left[s_{p+1}, s_{p+2}, \ldots, s_{K}\right]^{T}$ denotes the set of signals already detected and canceled in previous stages with $\hat{\boldsymbol{s}}_{K-p}$ representing its estimate, the matrix

$$
\begin{aligned}
\boldsymbol{H}_{p} & =\left[\begin{array}{cccc}
h_{1,1} & h_{1,2} & \cdots & h_{1, p} \\
h_{2,1} & h_{2,2} & \cdots & h_{2, p} \\
\vdots & \vdots & \ddots & \vdots \\
h_{L, 1} & h_{L, 2} & \cdots & h_{L, p}
\end{array}\right] \\
& =\left[\begin{array}{llll}
\boldsymbol{h}_{p, 1} & \boldsymbol{h}_{p, 2} & \cdots & \boldsymbol{h}_{p, p}
\end{array}\right]
\end{aligned}
$$

is an $L \times p$ matrix containing the channel coefficients of the $p$ remaining signals with estimate $\hat{\boldsymbol{H}}_{p}$, where we have denoted the $i$ th column of the channel matrix $\boldsymbol{H}_{p}$ by the vector $h_{p, i}$

$$
\boldsymbol{H}_{K-p}=\left[\begin{array}{cccc}
h_{1, p+1} & h_{1, p+2} & \cdots & h_{1, K} \\
h_{2, p+1} & h_{2, p+2} & \cdots & h_{2, K} \\
\vdots & \vdots & \ddots & \vdots \\
h_{L, p+1} & h_{L, p+2} & \cdots & h_{L, K}
\end{array}\right]
$$

is an $L \times(K-p)$ matrix containing the channel coefficients of the $K-p$ canceled signals, and $\hat{\boldsymbol{H}}_{K-p}$ is its estimate. 


\section{B. Signal Combining}

Using the channel-estimation model described in the previous section, (10) can be rewritten as

$$
\boldsymbol{r}_{p}=\boldsymbol{H}_{p} \boldsymbol{s}_{p}+\boldsymbol{\Upsilon}_{K-p} \boldsymbol{s}_{K-p}+\boldsymbol{j}_{p}+\boldsymbol{n}
$$

where $\boldsymbol{j}_{p}=\hat{\boldsymbol{H}}_{K-p}\left[\boldsymbol{s}_{K-p}-\hat{\boldsymbol{s}}_{K-p}\right]$ is the interference from previous stages due to detection errors and

$$
\begin{aligned}
\boldsymbol{\Upsilon}_{K-p} & =\boldsymbol{H}_{K-p}-\hat{\boldsymbol{H}}_{K-p} \\
& =-\left[\begin{array}{cccc}
\varepsilon_{1, p+1} & \varepsilon_{1, p+2} & \cdots & \varepsilon_{1, K} \\
\varepsilon_{2, p+1} & \varepsilon_{2, p+2} & \cdots & \varepsilon_{2, K} \\
\vdots & \vdots & \ddots & \vdots \\
\varepsilon_{L, p+1} & \varepsilon_{L, p+2} & \cdots & \varepsilon_{L, K}
\end{array}\right]
\end{aligned}
$$

is the channel-estimation error. Note that with channel-estimation errors, there is no perfect cancellation even with successful signal detection in the previous stages, i.e., when $\boldsymbol{s}_{K-p}=\hat{\boldsymbol{s}}_{K-p}$.

At stage $p$, the best signal for detection among the $p$ remaining signals is identified and then detected. This identification is done by first calculating a set of combining weights for each signal based on $\hat{\boldsymbol{H}}_{p}$ and then making the selection of the signal to be detected. The $p$ combined signal samples obtained at this stage can be written as

$$
\begin{aligned}
y_{p, k} & =\boldsymbol{\omega}_{p, k}^{H} \boldsymbol{H}_{p} \boldsymbol{s}_{p}+\boldsymbol{\omega}_{p, k}^{H} \boldsymbol{\Upsilon}_{K-p} \boldsymbol{s}_{K-p}+\boldsymbol{\omega}_{p, k}^{H} \boldsymbol{j}_{p}+\boldsymbol{\omega}_{p, k}^{H} \boldsymbol{n} \\
& =\boldsymbol{\omega}_{p, k}^{H} \boldsymbol{h}_{p, k} s_{p, k}+\sum_{i=1, i \neq k}^{p} \boldsymbol{\omega}_{p, k}^{H} \boldsymbol{h}_{p, i} s_{p, i}+j_{p, k}+z_{p, k}
\end{aligned}
$$

where $1 \leq k \leq p$ and $\boldsymbol{\omega}_{p, k}$ are the combining weight optimized for signal $k$ in detection stage $p$

$$
z_{p, k}=\boldsymbol{\omega}_{p, k}^{H} \boldsymbol{\Upsilon}_{K-p} \boldsymbol{s}_{K-p}+\boldsymbol{\omega}_{p, k}^{H} \boldsymbol{n}
$$

represents the total noise component, and $j_{p, k}=\boldsymbol{\omega}_{p, k}^{H} \boldsymbol{j}_{p}$ and $(\cdot)^{H}$ denote the conjugate transpose matrix. As $\varepsilon_{l, k}$ is zero-mean complex Gaussian with variance $N_{0} /\left(J A_{k}^{2}\right)$, the product $\varepsilon_{l, k} s_{p, k}$ is also zero-mean complex Gaussian with variance $N_{0} / J$. Thus, with uncorrelated signals $s_{p, k}, z_{p, k}$ is a zero-mean complex Gaussian random variable with variance given by

$$
\sigma_{z_{p, k}}^{2} \stackrel{\text { def }}{=} N_{0}^{\prime}=\left|\boldsymbol{\omega}_{p, k}\right|^{2}\left(\frac{K-p}{J}+1\right) N_{0}
$$

Note that the signal-to-noise ratio (SNR) degradation increases for each successive signal to be detected and is largest for the last signal to be detected. We can also see that perfect channel estimation is obtained when $J \mapsto+\infty$.

\section{Performance Analysis}

Consider again receiver stage $p$ and let us assume that $s_{p, m}$ has been selected for detection, with $1 \leq m \leq p$. Using (15), the signal sample corresponding to this signal can be written as

$$
\begin{array}{r}
y_{p} \stackrel{\text { def }}{=} y_{p, m}=\sqrt{E_{p, m}} e^{j\left(a_{p, m} \frac{2 \pi}{M}\right)}+\sum_{i=1, i \neq m}^{p} \sqrt{E_{p, i}} e^{j \phi_{p, i}} \\
+j_{p, m}+z_{p, m}
\end{array}
$$

where $E_{p, i}=\left|\boldsymbol{\omega}_{p, m}^{H} \boldsymbol{h}_{p, i}\right|^{2} E_{i}$ is the received symbol energy of signal $i$ after combining and $\phi_{p, i}$ is its phase $j_{p, m}$ in the interference from the previous stages due to error propagation.

For a given burst, the conditional SEP (given that no errors occurred in the previous stages) can be written as

$$
\begin{aligned}
P_{p}^{s}\left(\mathcal{E} \mid E_{p, i}, \phi_{p, i}\right) \approx Q\left(\sqrt{\frac{2 D_{1}^{2}}{N_{0}^{\prime}}}\right) & \\
+ & \left(a_{d}-1\right) Q\left(\sqrt{\frac{2 D_{2}^{2}}{N_{0}^{\prime}}}\right)
\end{aligned}
$$

for $p \geq 1$, where $Q(\lambda)=(1 / \sqrt{2 \pi}) \int_{\lambda}^{\infty} e^{-\frac{\lambda^{2}}{2}} d \lambda$.

$$
D_{1}=\sqrt{E_{p, m}} \sin \left(\frac{\pi}{M}\right)-\sum_{i=1, i \neq m}^{p} \sqrt{E_{p, i}} \sin \left(\phi_{p, i}-\frac{\pi}{M}\right)
$$

and

$$
D_{2}=\sqrt{E_{p, m}} \sin \left(\frac{\pi}{M}\right)+\sum_{i=1, i \neq m}^{p} \sqrt{E_{p, i}} \sin \left(\phi_{p, i}+\frac{\pi}{M}\right)
$$

are the distances to the decision thresholds for the signal to be detected, which for a symbol state at angle $\phi$ are given by the lines departing from the origin with angles $\phi \pm \frac{\pi}{M}$ and $a_{d}$ is related to the modulation level $M$ and is given by [14]

$$
a_{d}= \begin{cases}1, & \text { for BPSK } \\ 2, & \text { for higher modulation levels }(M>2)\end{cases}
$$

The average conditional SEP, $P_{p}^{s}$, is then obtained by averaging (19) over all possible values of $\phi_{p, i} \mathrm{~s}$, as represented by all possible combinations of data symbols for the signals $p \neq m$.

The relation between the conditional and average SEP for SC-MUD of narrow-band signals in an AWGN channel, which takes into account error propagation, has been derived in [3, Eq. (12)]. In this model, the partial suppression or even enhancement of the signal after cancellation due to an error is assumed to result in random symbol detection for the signals that still need to be detected, which is a worst-case assumption. Since the radio channel is assumed constant over one burst, the SEP of the SC-MUD over one burst in Rayleigh-fading channels will also take the form of [3, Eq. (12)]. By taking the average over 


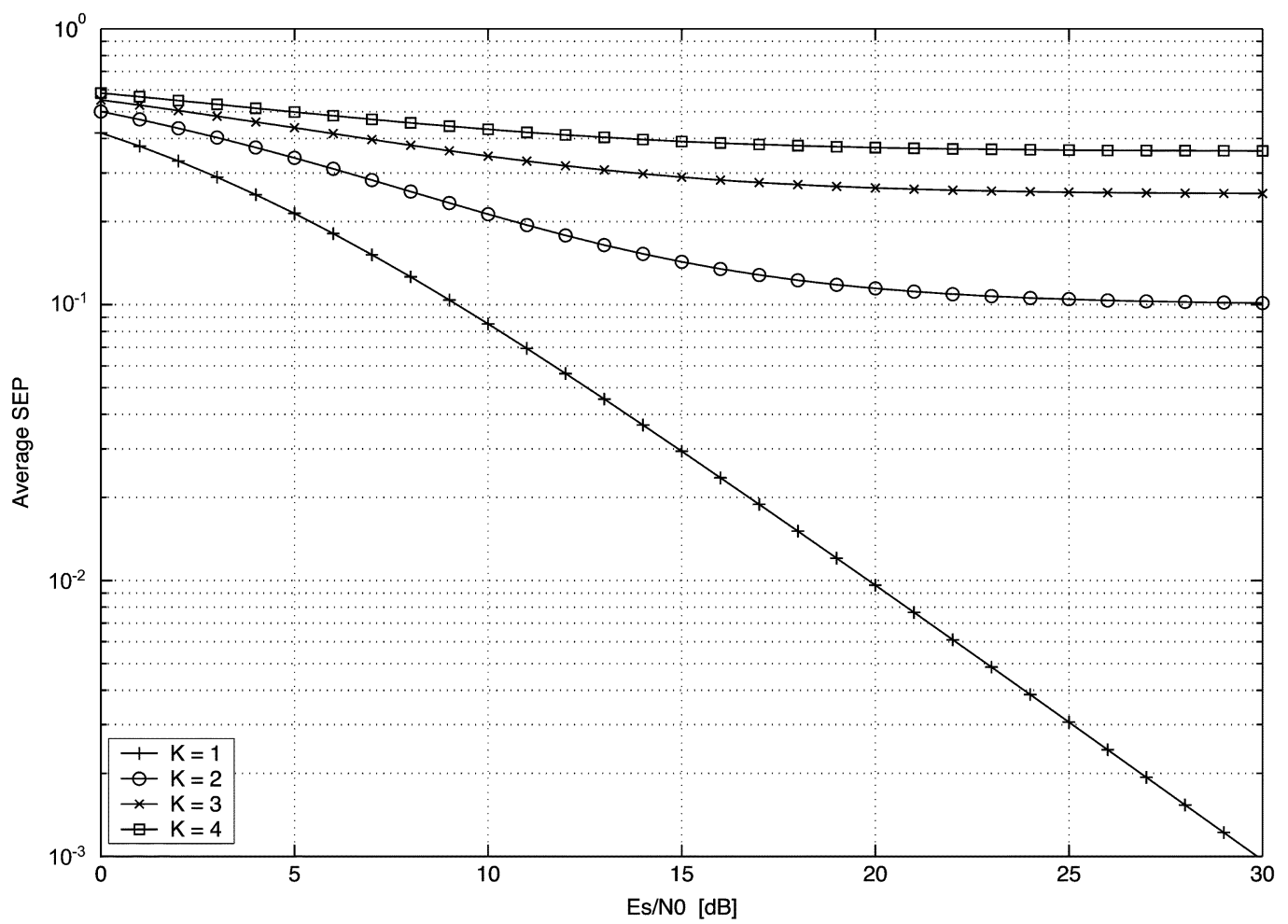

Fig. 2. Average SEP of the optimum SC-MUD for $K=1, \ldots, 4$ users with equal average received power in Rayleigh-fading channels.

all possible channel states, we obtain the average SEP that accounts for error propagation and channel variations.

\section{Single-Antenna Case}

For the single-antenna case, there is no redundancy and the receiver can only rely on the power difference between the different signals. In this case, the signal at stage $p$ is directly obtained from (15) with

$$
y_{p, k}=\left|h_{k}\right| s_{p, k}+\sum_{i=1, i \neq k}^{p} h_{i} e^{-j \theta_{k}} s_{i}+j_{p, k}+z_{p, k}
$$

where $1 \leq k \leq p, h_{i}$ is the channel coefficient between user $i$ and the receiver and $z_{p, k}$ is zero-mean complex Gaussian with variance $\sigma_{z_{p, k}}^{2}=\sigma_{z_{p}}^{2} \stackrel{\text { def }}{=} N_{0}^{\prime}=N_{0}[1+(K-p) / J]$. The noise variance is independent of $k$ because the combining weight is just the phase rotation of the fading coefficient of the user to be detected, $\omega_{k}=e^{-j \theta_{k}}$. Since the fading channel is assumed to be constant over the burst duration, the detection order of the signals can be identified at the first stage. Of course, depending on the channel variation, this order may be different from one burst to the next.

Taking into account signal ordering, (20) can be rewritten in the same form as (18). This ordering provides us with an optimum SC-MUD, but makes a closed form for the average SEP difficult to obtain due to the order statistics involved. Fig. 2 shows the average SEP for the optimum receiver and multiple users with equal average received powers, as obtained by simulations for quaternary phase-shift keying (QPSK) modulation.
These results clearly show the very poor symbol error rate performance when more than one user are sharing the same bandwidth. For $K>1$, error floors occur due to the large interference levels at the different stages caused by signal fading and error propagation. Thus, when no diversity scheme is employed, the single-antenna case requires extra power margins between the different users to ensure reliable communication for all the links simultaneously. By controlling the transmitted power of the different users, we can improve the SEP of the different users. The occurrence of the decoding order at the receiver will depend on the selected power margins between the different signals. In this case, instead of the optimum receiver, we may consider a hypothetical suboptimum SC-MUD, where a closed-form expression for its average SEP can be obtained. To accomplish that, we assume that the receiver detects the signals in decreasing order of their received average symbol energies $\bar{E}_{k}=E_{k} \overline{\left|h_{k}\right|^{2}}=E_{k}$.

Basically, the suboptimum receiver considers the long-term average symbol energy and does not take into account the instantaneous channel coefficients when ordering the signals. During a burst, a weaker signal may be detected before a larger one, which results in a poorer SEP when compared to the optimum SC-MUD. Thus, the SEP performance of the suboptimum receiver is an upper bound for the SEP of the optimum SC-MUD.

Using (20) and modeling the interference as Gaussian, the conditional SEP (given that no error has occurred in the previous stages) for the suboptimum SC-MUD, is approximated as [14]

$$
P_{k}^{s} \approx \frac{a_{d}}{2}\left[1-\sqrt{\frac{\beta \gamma_{k}}{1+\beta \gamma_{k}}}\right]
$$




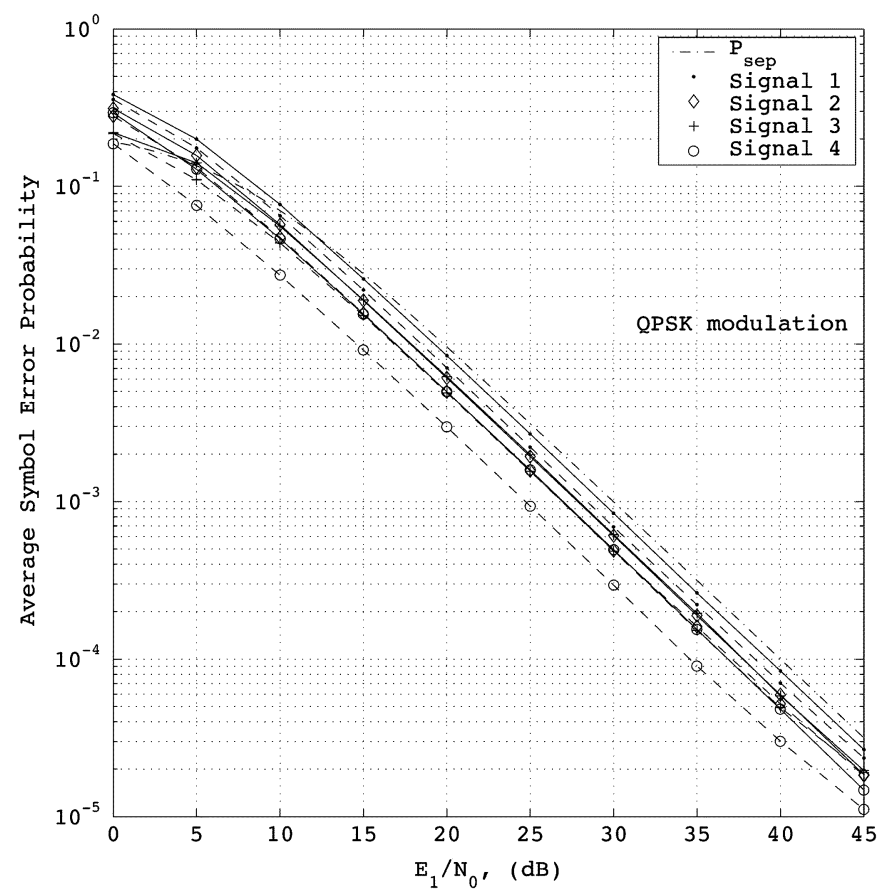

Fig. 3. Average SEP of the suboptimum (solid line) and optimum (dashed line) SC-MUD in Rayleigh-fading channels with controlled power allocation for the different users; $P_{\text {sep }}$ is the target SEP.

where $\beta=\sin ^{2}(\pi / M)$ and $\gamma_{k}$ are the expected received signal-to-noise-plus-interference ratio (SNIR) of signal $s_{k}$ and is given by

$$
\gamma_{k}=\frac{E_{k}}{\sum_{i=1, i \neq k}^{p} E_{i}+N_{0}^{\prime}} .
$$

Evaluation by simulations shows that the average SEP of the suboptimum receiver is less than twice the SEP of the optimum SC-MUD. Using the above expression and following the same procedure as described in [3], the required power margins between the different signals that ensure a target SEP $P_{\text {sep }}$ for the different users can be derived. Without going into detail, but assuming that $E_{K}>E_{K-1}>\cdots>E_{1}$, these power margins are given by

$$
E_{k}=\left[\frac{G\left(P_{\mathrm{sep}}, M, k\right)}{\sin ^{2}\left(\frac{\pi}{M}\right)}+\frac{G\left(P_{\mathrm{sep}}, M, k\right)}{G\left(P_{\mathrm{sep}}, M, k-1\right)}\right] E_{k-1}
$$

where $2 \leq k \leq K$

$$
G\left(P_{\mathrm{sep}}, M, k\right)=\frac{1}{1-\left(1-2 P_{k}^{s}\left(P_{\mathrm{sep}}, M\right) / a_{d}\right)^{2}}-1
$$

and $P_{k}^{s}\left(P_{\text {sep }}, M\right)$ is as defined in [3, Eq. (24)].

To illustrate the performance of the single-antenna SC-MUD in the Rayleigh-fading channel, we consider a system with $K=$ 4 users employing QPSK modulation and apply the above derived power margins. Fig. 3 gives simulation results for the SEP of the suboptimum and the optimum SC-MUD as a function of $E_{1} / N_{0}$. Also included in this figure is the target (analytical) SEP. It is shown that the SEPs of the different signals are similar and are quite close to the target SEP. We observe that the $o p$ timum SC-MUD performs slightly better than the suboptimum

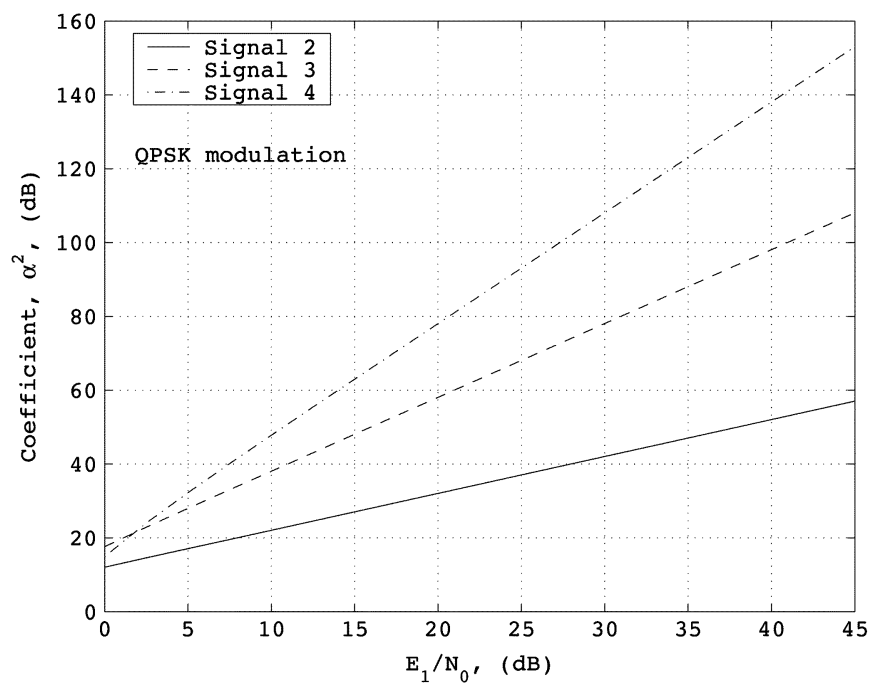

Fig. 4. Required power margins for the suboptimum SC-MUD detector that achieve the same target SEP for each of the signals in Rayleigh-fading channels.

SC-MUD. Thus, by using the above power margins, it is possible to provide a preset SEP for the different signals. Also, these results suggest that the above analysis for the suboptimum detector can be used as a tight upper bound on the performance of the optimum SC-MUD for SEP levels of practical interest, where the probability of a wrong detection order is small. Fig. 4 shows the power margins in decibels as a function of $E_{1} / N_{0}$. We notice that these power margins are linearly related to the SNR of the weakest signal and that, in order to achieve acceptable SEP, very high power margins are required. Increasing the number of users will increase these power margins even more.

\section{Signal-SELECTION AND COMBINING SCHEMES}

By applying antenna diversity at the receiver, the system performance in the fading channel can be improved. The scenario for the SC-MUD, however, is different from conventional scenarios in which a single desired signal is detected in the presence of other interfering signals and noise, since here we want to detect multiple desired signals in the presence of noise. In the SC-MUD, the conditional SEP of the signal detected at a certain stage not only determines the detection performance of this signal, but also that of the other not-yet-detected signals due to error propagation. Therefore, the antenna-combining scheme and the order of detection have to be matched to this behavior. This means that at each detection stage the following steps are needed:

- determine the weight vector optimized to the signal scenario in this stage with the not-yet-detected signals present;

- select the signal that, after combining, is likely to result in the lowest conditional SEP, in order to minimize error propagation;

- detect the selected signal and cancel it from the received signal.

In the following, the detection order and combining weights are determined from the per-burst estimated channel parameters. 


\section{A. Order of Detection}

As indicated in (19), the conditional SEP depends on the distance between the resulting compound state from all signals present and the decision threshold, as well as on the noise term. Therefore, choosing the signal (and corresponding weight vector) that results in the largest SNR does not guarantee the best detection quality, as in the one-signal case. Two criteria are used to select the best signal for detection in stage $p$, which take into account the interfering signals but assume perfect cancellation of the already-detected signals.

1) Maximizing SNIR: The SNIR presents a good indication for the detection performance. Here, we select the signal that maximizes the SNIR in detection stage $p$. For signal $s_{p, k}$, the SNIR is given by

$$
\Gamma_{p, k}=\frac{\left|\boldsymbol{\omega}_{p, k}^{H} \boldsymbol{h}_{p, k} s_{p, k}\right|^{2}}{\sigma_{z_{p, k}}^{2}\left|\boldsymbol{\omega}_{p, k}\right|^{2}+\sum_{\substack{i=1 \\ i \neq k}}^{p}\left|\boldsymbol{\omega}_{p, k}^{H} \boldsymbol{h}_{p, i} s_{p, i}\right|^{2}}
$$

where $\boldsymbol{\omega}_{p, k}$ is the weight vector optimized for $s_{p, k}$.

The desired signal $m$, which has the maximum estimated SNIR

$$
\hat{\Gamma}_{p, m}=\max _{k}\left\{\hat{\Gamma}_{p, k}\right\}
$$

is selected for detection. For selection we use the estimated SNIR $\hat{\Gamma}_{p, k}$, which is obtained from (25) by replacing $\boldsymbol{h}_{p, i}$ with $\hat{\boldsymbol{h}}_{p, i}$. This criterion is indicated as maximizing the SNIR (MaxSNIR). Note that MaxSNIR does not necessarily maximize the distance to the detection threshold.

2) Maximizing the Minimum Distance-to-Noise Ratio: The highest conditional SEP occurs when the compound state of the signals present in the current stage $p$ is at the minimum distance to the decision threshold (worst case). By maximizing the minimum distance-to-noise ratio (DNR) defined by

$$
D_{p, k} \triangleq \frac{\left|\boldsymbol{\omega}_{p, k}^{H} \boldsymbol{h}_{p, k} s_{p, k}\right| \sin \left(\frac{\pi}{M}\right)-\sum_{\substack{i \neq 1 \\ i \neq k}}^{p}\left|\boldsymbol{\omega}_{p, k}^{H} \boldsymbol{h}_{p, i} s_{p, i}\right|}{\sigma_{z_{p, k}}\left|\boldsymbol{\omega}_{p, k}\right|}
$$

we minimize the worst case conditional SEP. The signal $m$, which has maximum estimated DNR

$$
\hat{D}_{p, m}=\max _{k}\left\{\hat{D}_{p, k}\right\}
$$

is selected for detection, where the estimated DNR $\hat{D}_{p, k}$ is obtain from (27) by replacing $\boldsymbol{h}_{p, i}$ with $\hat{\boldsymbol{h}}_{p, i}$. This selection criterion is called the MaxDNR criterion.

\section{B. Combining Schemes}

As combining schemes, we consider ZF and mmse combining. The combining weights are determined from the estimated channel coefficients. Interference caused by occasional erroneous signal cancellation due to symbol-estimation errors is not taken into account in the weight calculation. Schemes that do not actively reduce interference, such as maximal ratio combining and selection diversity, perform very poorly when interference is present and, therefore, are not further considered here.

1) $Z F$ : With $\mathrm{ZF}$, the contributions of the interfering signals are forced to zero, if possible. In general, this is achieved at the cost of noise amplification. To find the weight vector for signal $s_{p, k}$ in stage $p$, we impose the ZF condition on the estimated channel coefficients

$$
\hat{\boldsymbol{H}}_{p}^{H} \boldsymbol{\omega}_{p, k}=\boldsymbol{c}
$$

where $\mathbf{c}$ is a vector of length $p$ with all zeros except for a 1 in the $k$ th position. Instead of the inverse $\hat{\boldsymbol{H}}_{p}^{-1}$, we use the Moore-Penrose pseudoinverse $\hat{\boldsymbol{H}}_{p}^{\dagger}[15]$ to determine the weight vector $\boldsymbol{\omega}_{p, k}=\left(\hat{\boldsymbol{H}}_{p}^{H}\right)^{\dagger} \boldsymbol{c}$. By using the pseudoinverse, we obtain

1) least-squared Euclidean distance between $\hat{\boldsymbol{H}}_{p}^{H} \boldsymbol{\omega}_{p, k}$ and $c$ when $L<p$ and the linear system (29) is underdetermined;

2) unique solution $\boldsymbol{\omega}_{p, k}=\left(\hat{\boldsymbol{H}}_{p}^{H}\right)^{-1} \boldsymbol{c}$ when $\hat{\boldsymbol{H}}_{p}$ is invertible;

3) ZF solution with minimum $\left|\boldsymbol{\omega}_{p, k}\right|$ when $L>p$, which results in least noise amplification.

The best signal to detect is the one with the smallest conditional SEP. In case $L<p$, some residual interference is left and the estimated SNIR is found with (25). For 2) and 3), all interference is canceled and (25) turns into the estimated SNR given by

$$
\hat{\Gamma}_{p, k}=\frac{\left|\boldsymbol{\omega}_{p, k}^{H} \hat{\boldsymbol{h}}_{p, k} s_{p, k}\right|^{2}}{\sigma_{z_{p, k}}^{2}\left|\boldsymbol{\omega}_{p, k}\right|^{2}}=\frac{A_{p, k}^{2}}{\sigma_{z_{p, k}}^{2}\left|\boldsymbol{\omega}_{p, k}\right|^{2}}
$$

2) mmse: The mmse criterion [16] maximizes the SNIR. The advantage of mmse over $\mathrm{ZF}$ is that the noise power is taken into account. With $\boldsymbol{\omega}_{p, k}$, the error signal after combining is given by

$$
\begin{aligned}
\xi_{p, k} & =\boldsymbol{\omega}_{p, k}^{H}\left(\boldsymbol{r}_{p}-\hat{\boldsymbol{h}}_{p, k} s_{p, k}\right) \\
& =\boldsymbol{\omega}_{p, k}^{H} \boldsymbol{r}_{p-1}^{\prime}
\end{aligned}
$$

where $\boldsymbol{r}_{p}$ is given by (10) with $\boldsymbol{j}_{p}=0$, since error propagation due to decision errors is not taken into account in determining the weight vector. Notice that $\boldsymbol{r}_{p-1}^{\prime}$ becomes $\boldsymbol{r}_{p-1}$ in case $\boldsymbol{s}_{p, k}$ is detected in stage $p$. Thus, we can write the received interference vector as $\boldsymbol{x}=\boldsymbol{H}_{p-1}^{\prime} \boldsymbol{s}_{p-1}^{\prime}$ and the noise vector as $\boldsymbol{z}=\boldsymbol{\Upsilon}_{K-p+1}^{\prime} \boldsymbol{s}_{K-p+1}^{\prime}+\boldsymbol{n}$ and form the $L \times L$ interference and noise covariance matrices as $\boldsymbol{R}_{x}=E\left\{\boldsymbol{x} \boldsymbol{x}^{H}\right\}$ and $\boldsymbol{R}_{z}=E\left\{z z^{H}\right\}=\sigma_{z}^{2} \boldsymbol{I}$. The optimum weight vector $\boldsymbol{\omega}_{p, k}$, which minimizes the power in the error signal $\xi_{p, k}$ is obtained by [17]

$$
\boldsymbol{\omega}_{p, k}=\left(\boldsymbol{R}_{x}+\sigma_{z}^{2} \boldsymbol{I}\right)^{-1} \hat{\boldsymbol{h}}_{p, k}
$$

which is the Wiener solution. The SNIR after combining is again found with (25).

\section{Simulation Results}

The performance of the SC-MUD with antenna combining has been assessed using Monte Carlo simulations. The SEP 


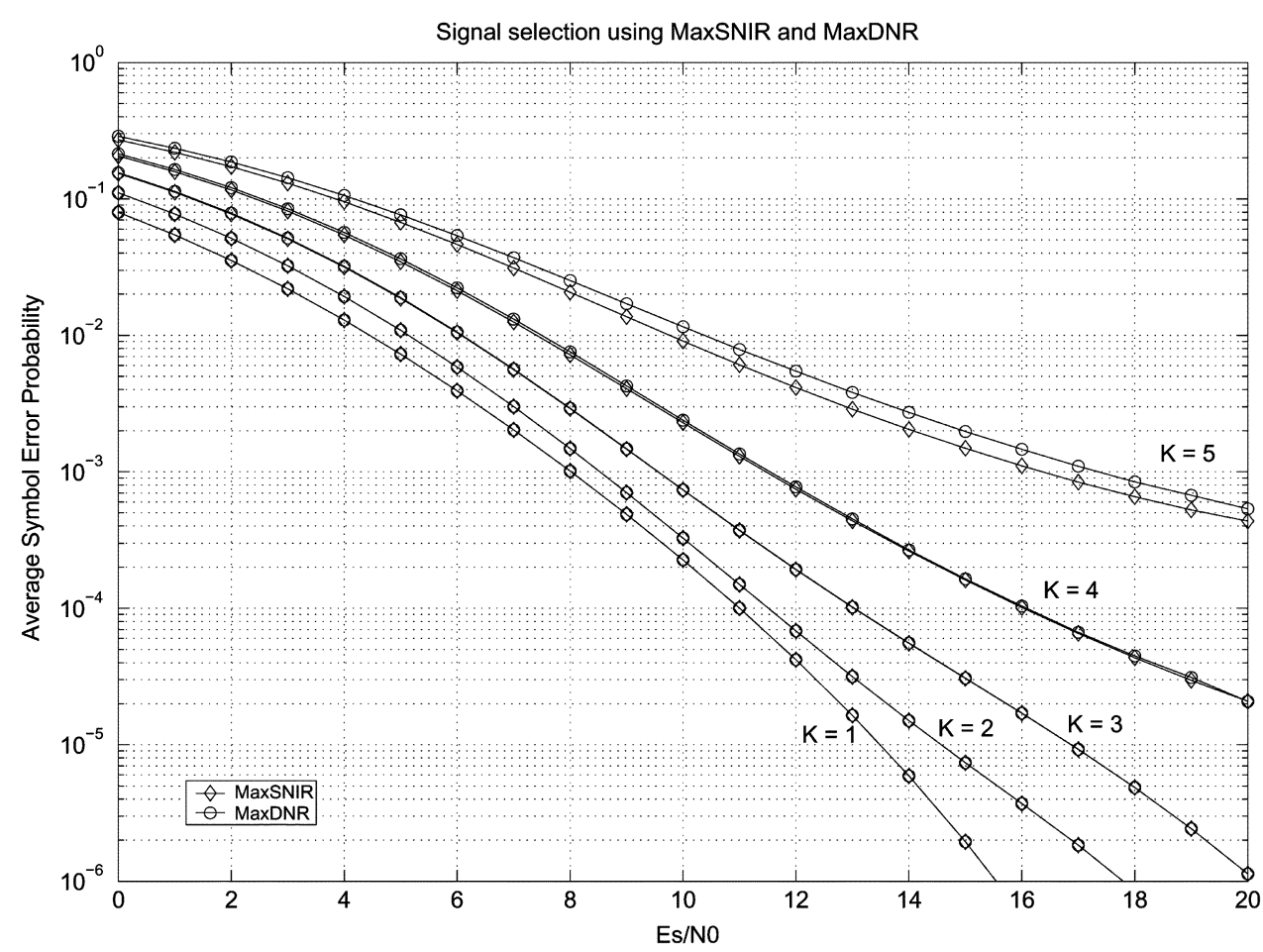

Fig. 5. SEP for MaxSNIR and MaxDNR signal selection for QPSK modulation, mmse combining with $L=4$ antennas, and the number of users $K$ as a parameter. All users have the same average received power.

was obtained from a large number of events (typically 40000 ), where in each event i.i.d. Rayleigh-distributed channel amplitudes for the users were generated and a word of 100 random symbols per user was transmitted for estimating the average conditional SEP, as indicated by (19). Unless indicated otherwise, perfect channel estimation is assumed. To determine the SEP of a single user over many independent channel events, the detection order of this user was tracked for each event to account for the error propagation.

We compare signal-selection criteria and combining schemes and evaluate the effects of the number of users, the number of antennas, user signal power differences, and channel-estimation errors.

\section{A. Signal Selection}

In each stage of the SC-MUD, we select the signal to be detected as the one that will most likely result in the lowest conditional SEP. In Fig. 5, the performances of the MaxSNIR and the MaxDNR criteria are compared. In this figure, the average SEP is shown for QPSK-modulated signals with mmse combining using $L=4$ antennas. We observe that both schemes perform equally well for $K \leq L$. Only for $K>L$, MaxSNIR slightly outperforms MaxDNR. For ZF, similar results are found. In the following, MaxSNIR is used for signal selection.

\section{B. Combining Schemes}

In Fig. 6, the average SEP is shown for QPSK modulation with $\mathrm{ZF}$ and mmse combining, as a function of the number of antenna elements $L$ with the number of users as a parameter. All users have the same average received power and the SNR has been fixed to $E_{s} / N_{0}=15 \mathrm{~dB}$. It is observed that both combining schemes result in considerable performance improvement as the number of antennas increases, i.e., antenna combining not only mitigates the deteriorating effects of channel fading, but also compensates for the interference that the signals are causing to each other. Actually, the SC-MUD is able to operate at low SEP without any power margins applied as required in the single antenna case. The mmse slightly outperforms ZF, since ZF results in noise amplification.

\section{SNR Penalty for Additional Users}

Fig. 7 shows upperbound SEP results for equi-power binary phase-shift keying (BPSK) and QPSK signals for the ML-JD receiver, as a function of $E_{s} / N_{0}$. Similar results are shown for the SC-MUD with mmse combining in Fig. 8. By comparing these results, we observe equal performance for the SC-MUD and ML-JD in the single-user case. By adding an extra user to the system, more interference is created and, thus, more power is required to obtain the same SEP. In [7] and [18], SNR penalties for additional users were analytically derived for ML-JD of narrow-band signals with respect to the modulation level $M$ and the number of antenna elements $L$. For BPSK modulation with two and four antennas, the penalties for an additional user are 0.5 and $0.1 \mathrm{~dB}$, respectively. For QPSK modulation, the penalty is $0.2 \mathrm{~dB}$ for the case of four antennas, as shown in Fig. 7. A similar behavior is observed for the SC-MUD in Fig. 8. These results show that an additional user can be added at the cost of a slightly higher $E_{s} / N_{0}$ than for ML-JD. It is observed that the SNR penalty is a fixed value for $K<L$ and for moderate values of $E_{s} / N_{0}$ : about 1 and $0.5 \mathrm{~dB}$ for BPSK with two and four antennas, respectively, and $1 \mathrm{~dB}$ for QPSK with four antennas. The penalty increases for $K \geq L$ as 


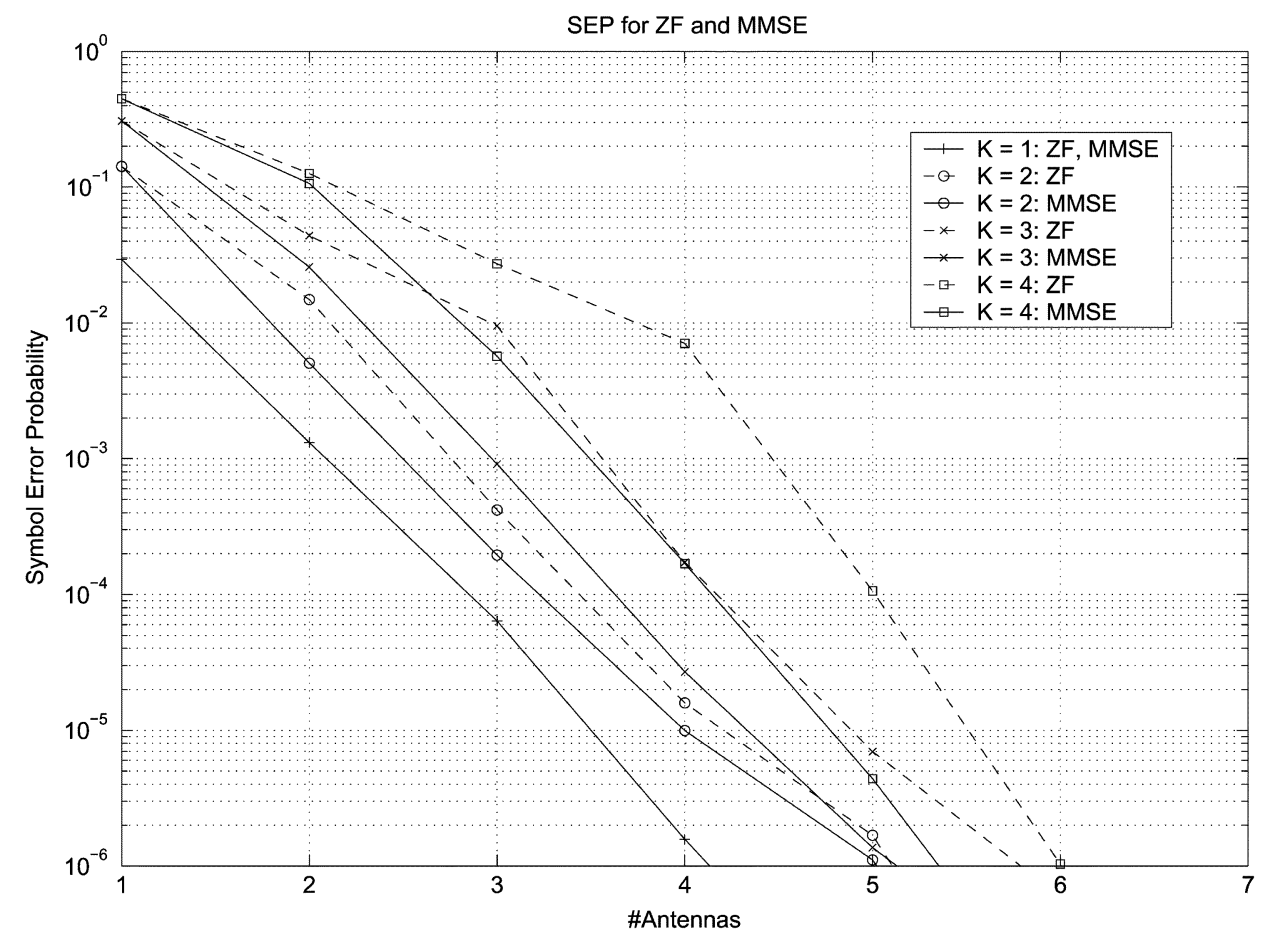

Fig. 6. SEP for QPSK as a function of the number of antennas for ZF and mmse with the number of users $K$ as a parameter. The average received power of the different users is the same with $E_{s} / N_{0}=15 \mathrm{~dB}$.

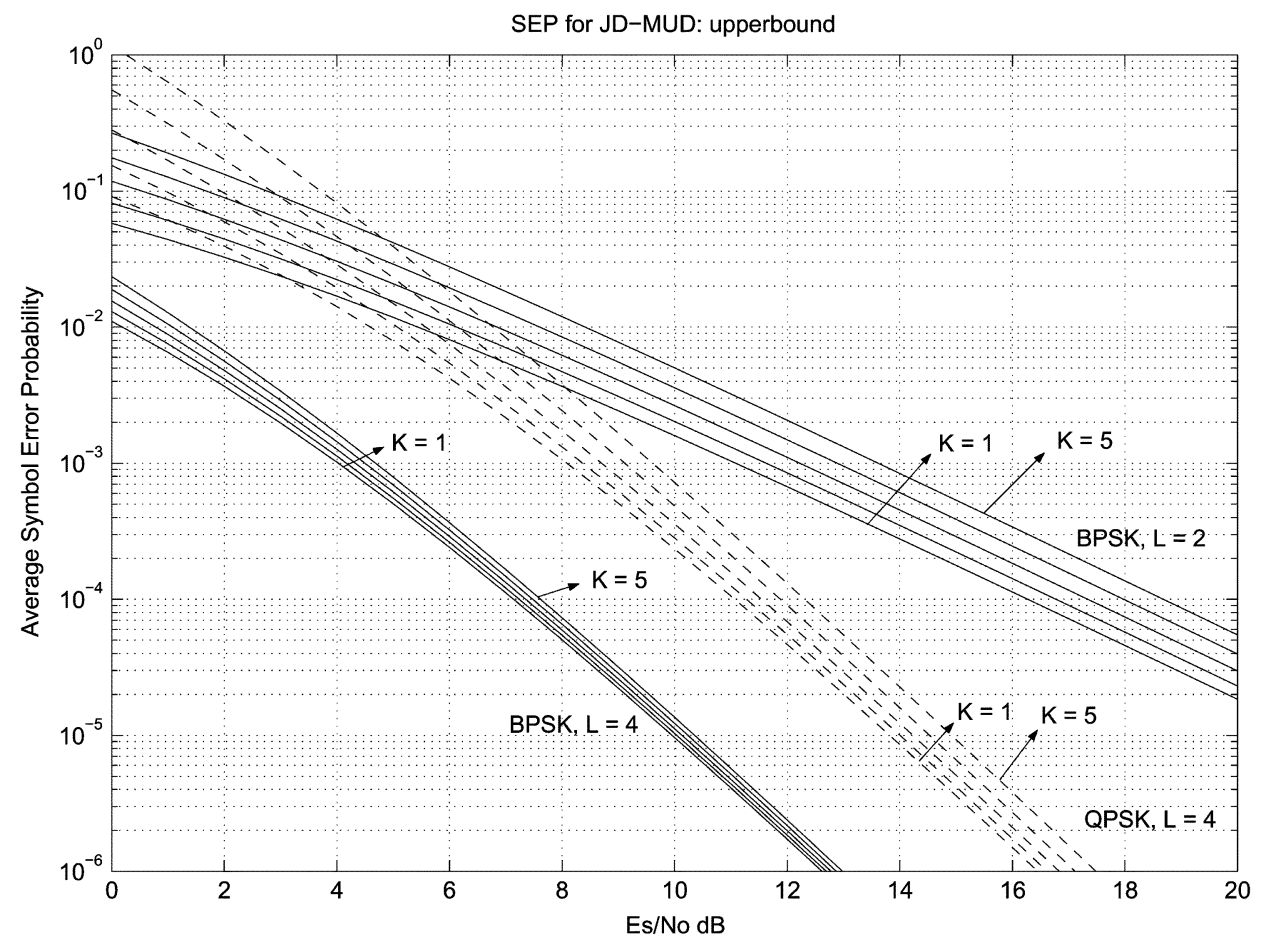

Fig. 7. Upper bound results [7], [18] for the SEP as a function of $E_{s} / N_{0}$ for JD-MUD in the Rayleigh-fading channel for binary phase-shift keying (BPSK) with $\mathrm{L}=2,4$ antennas and QPSK with $\mathrm{L}=4$ antennas and $\mathrm{K}=1-5$ users.

well as for high $E_{s} / N_{0}$. One reason for the additional performance degradation of the SC-MUD with increasing $K$ is error propagation in the multistage detector. The observed degradation for high $E_{s} / N_{0}$ as well as the progressive degradation for $K \geq L$ is due to the remaining error signal after combining, which increases, especially for $K \geq L$, because mmse is less able to reduce the interference if the number of signals be- comes larger. In a practical implementation, this would mean that the base station has to be equipped with a number of antennas that is at least equal to or larger than the number of users that is expected to be served. However, if the number of users $K \geq L$, the degradation can be compensated for by introducing small power differences between the signals, as is shown in the following. 

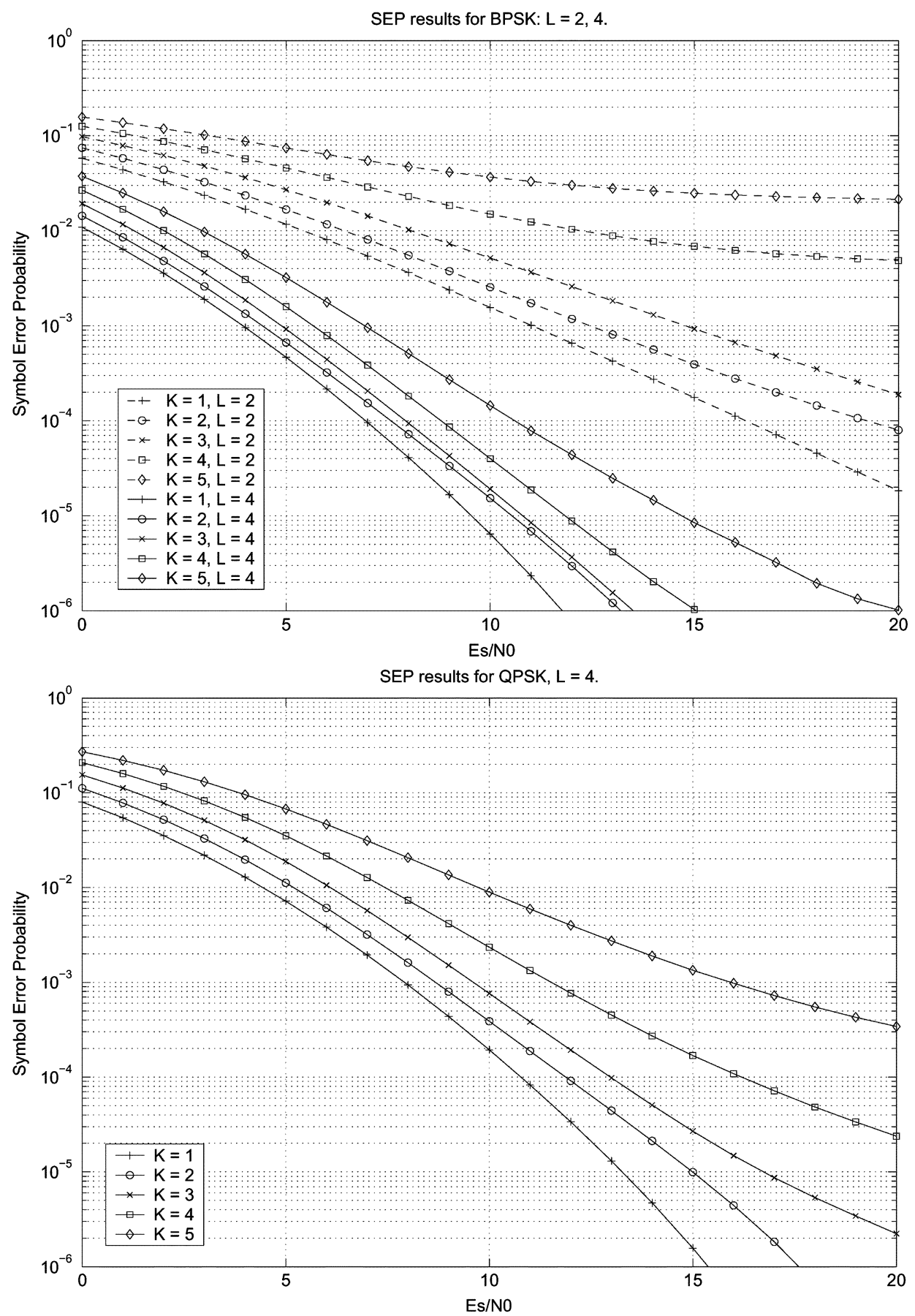

Fig. 8. SEP as a function of $E_{s} / N_{0}$ with the number of users $K$ as a parameter for BPSK and QPSK and a different number of antennas. The mmse combining is used and all users have equal average received power.

\section{Application of Small Power Margins}

Power disparity between users is expected to improve SC-MUD performance. To assess this effect, we have assumed that the average received symbol energies of the different users are geometrically related with $E_{k}=\alpha^{2(k-1)} E_{1}$ with $\alpha \geq 1$, so signal 1 is the weakest received signal. Fig. 9 shows the average SEP of the different users as a function of $\alpha$ with the number of antennas as a parameter and $E_{1} / N_{0}=15 \mathrm{~dB}$. It is observed that a substantial improvement in the performance can be obtained by introducing small power margins. The relative improvement increases with increasing the number of antennas.
It is also observed that for small $\alpha$, the obtained improvement is almost the same for all signals. This is because, for small $\alpha$, the average SEP is dominated by error propagation, which is reduced when $\alpha$ is increased. For large $\alpha$, the signals become more separated and the effect of error propagation on the SEP of the smaller signals becomes negligible.

\section{E. Effect of Channel-Estimation Errors}

In the case of channel-estimation errors, the noise level increases slightly due to cancellation errors for each successive 


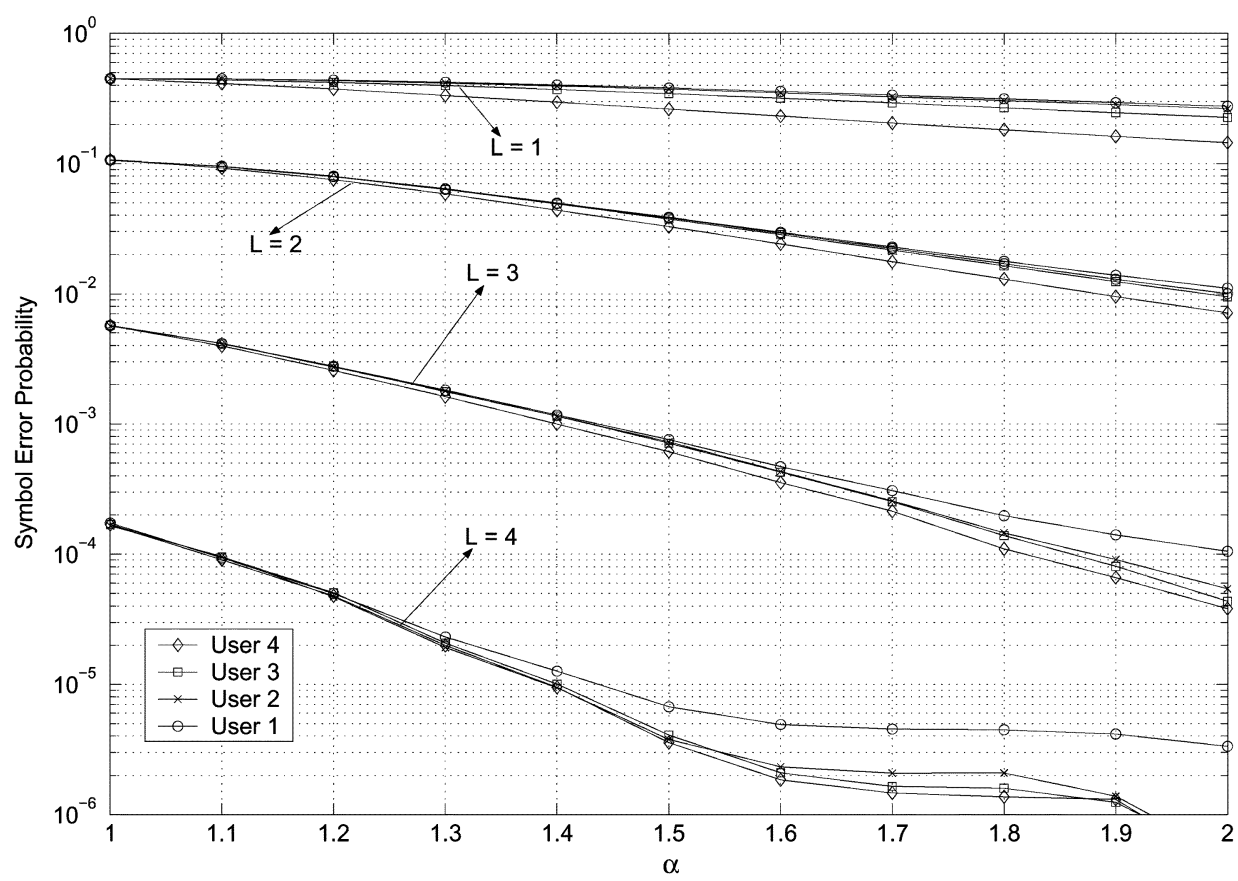

Fig. 9. SEP for QPSK modulation as a function of the amplitude ratio $\alpha$ and the number of antennas as a parameter. The mmse combining is employed and $E_{s 1} / N_{0}=15 \mathrm{~dB}$.

signal to be detected. For the simulations, we introduce estimation errors according to (9). Fig. 10 shows the effect of estimation errors on the average SEP for equal-power users in a Rayleigh-fading channel for QPSK modulation with mmse and ZF combining. The performance for training sequences of length $J=16$ and $J=32$ are compared to that with perfect estimation, i.e., $J=+\infty$, with the number of users $K$ as a parameter. It is observed that the performance degradation due to estimation errors because of a limited training sequence length is rather small for both mmse and ZF combining. This degradation can be compensated for by a small increase in SNR.

\section{F. Discussion}

The results presented previously clearly show that a tradeoff can be made between the number of users $K$, the number of antennas $L$, and the power margins that are required, as well as $E_{s 1} / N_{0}$. The large power margins needed for $L=1$ can be reduced to no power margin at all by applying antenna diversity. For example, in Fig. 3 we find an average SEP of about $2 \cdot 10^{-2}$ for $K=4$ and $L=1$ at $E_{s 1} / N_{0}=15 \mathrm{~dB}$ and power margins between 26 and $63 \mathrm{~dB}$. In Fig. 6, we obtain a better SEP for $K=4$ and mmse with $L=3$ and $E_{s} / N_{0}=15 \mathrm{~dB}$ without any power margins at all. From Fig. 9, we observe that the same SEP can be obtained at $E_{s 1} / N_{0}=15 \mathrm{~dB}$ with $L=2$ antennas and equal power margins between the signals of about $6 \mathrm{~dB}$. Thus, by using $L=2$ antennas instead of a single antenna, the required power margins can be reduced dramatically.

\section{CONCLUSION}

An analytical model is presented to evaluate the performance of a narrow-band SC-MUD in the Rayleigh-fading channel for a single antenna and with antenna diversity. This model accounts for the effects of channel-estimation errors and detection-error propagation.

For the SC-MUD with a single antenna, two cases were compared: the optimum case (detection order based on instantaneous receive power levels) and the suboptimum case (detection order based on the average receive power levels); the required power margins for the different users to achieve a given preset SEP were derived. Simulation results show that the derived power margins are quite accurate and result in an SEP close to the preset value. However, the required margins are very high for the Rayleigh-fading channel and increase quickly with increasing number of users. The analytical SEP for the suboptimum SC-MUD forms a rather tight upper bound for the $o p$ timum SC-MUD's performance.

To take maximum advantage of antenna diversity in the fading channel, the signal to be detected as well as the corresponding combining weights are to be determined jointly in each detection stage of the SC-MUD. The two proposed criteria to select the best signal for detection in a certain stage are the MaxSNIR, based on maximizing the SNIR, and MaxDNR, based on maximizing the minimum distance-to-noise ratio, which show nearly equal performance. Antenna diversity using ZF and mmse combining has been evaluated and compared to the optimum ML-JD, which has a higher computational complexity than the SC-MUD. The obtained results show that $\mathrm{ZF}$ and mmse not only effectively mitigate the degrading effects of fading, but also perform well with much smaller or even without the need for power margins between the different signals as required in the single-antenna case. When the number of users $K$ is less than the number of antennas $L$, an extra user can be added at the cost of a small increase in SNR (dependent on the number of antennas and the modulation scheme applied). For mmse and $K<L$, this SNR penalty is about constant for moderate values of $E_{s} / N_{0}$, but slightly larger than the penalty 

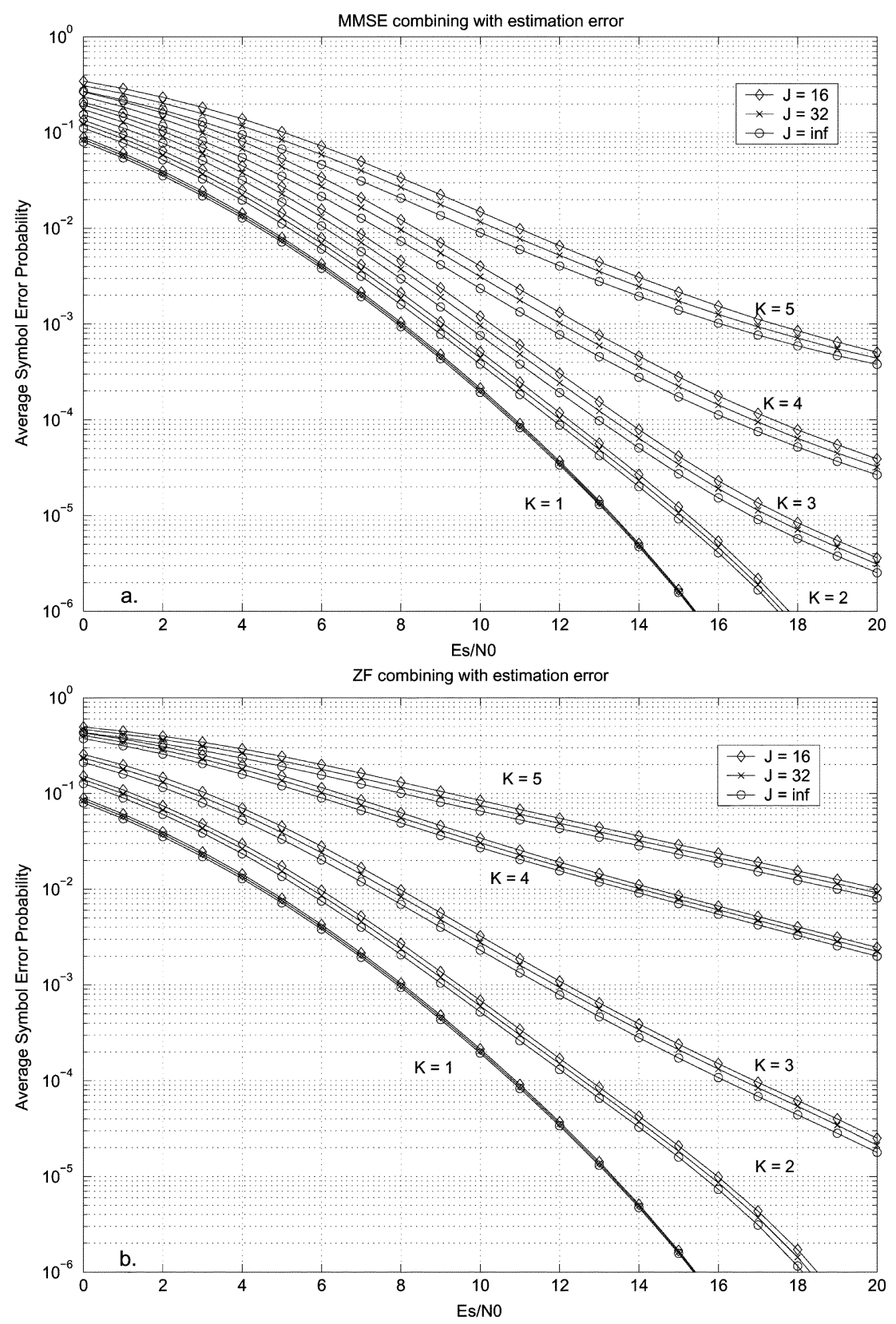

Fig. 10. SEP in the case of channel-estimation errors for different training sequence lengths $J$, with QPSK modulation, $L=4$, and for (a) mmse combining and (b) ZF combining. All users have the same average $E_{s} / N_{0}$.

required for the ML-JD [7]. For $K \geq L$, the penalty increases due to the remaining error signal after mmse combining. In a practical implementation, this would mean that the base station has to be equipped with a number of antennas larger or equal to the number of users that is expected to be served. However, for $K \geq L$ the degradation can also be compensated for by introducing (small) power margins between the signals.

The effect of channel-estimation errors on the SEP has been analyzed for short orthogonal training sequences (lengths 16 and 32). Simulation results for equal-power users in the Rayleigh-fading channel show that the resulting channel-estimation errors cause a limited performance degradation compared to perfect estimation. This degradation can be compensated for by a small increase in SNR.

\section{REFERENCES}

[1] S. Verdu, Multiuser Detection. Cambridge, U.K.: Cambridge Univ. Press, 1998.

[2] G. J. M. Janssen and S. Ben Slimane, "Symbol error probability of a M-PSK multiuser detector using successive cancellation," Electron. Lett., vol. 36, no. 25, pp. 2103-2105, 2000.

[3] — "Symbol error probability analysis of a multiuser detector for M-PSK signals based on successive cancellation," IEEE J. Select. Areas Commun., vol. 20, pp. 330-338, Feb. 2002.

[4] H. Arslan and K. Molnar, "Cochannel interference suppression with successive cancellation in narrow-band systems," IEEE Commun. Lett., vol. 5, pp. 37-39, Feb. 2001.

[5] B. Hagerman, "Single-user receivers for partly known interference in multi-user environments," Ph.D. dissertation, Radio Commun. Syst., Royal Inst. Technol., Stockholm, Sweden, Oct. 1995.

[6] R. Kwan and C. Leung, "Optimal detection of a BPSK signal contaminated by interference and noise," IEEE Commun. Lett., vol. 6, pp. 225-227, June 2002. 
[7] S. J. Grant and J. K. Cavers, "Performance enhancement through joint estimation of co-channel signal using diversity arrays," IEEE Trans. Commun., vol. 46, pp. 1039-1049, Aug. 1998.

[8] G. J. M. Janssen and J. Zander, "Capacity assessment of a cellular radio system using a narrowband multiuser detector," IEEE Trans. Wireless Commun., vol. 2, pp. 703-713, July 2003.

[9] P. W. Wolniansky, G. J. Foschini, G. D. Golden, and R. A. Valenzuela, "V-BLAST: An architecture for realizing very high data rates over the rich-scattering wireless channel," in Proc. Int. Symp. Signals Systems and Electronics (ISSSE'98), Oct. 1998, pp. 295-300.

[10] G. J. Foschini, G. D. Golden, R. A. Valenzuela, and P. W. Wolnianski, "Simplified processing for high spectral efficiency wireless communication employing multi-element arrays," IEEE J. Select. Areas Commun., vol. 17, pp. 1841-1852, Nov. 1999.

[11] H. L. van Trees, Detection Estimation and Modulation Theory. New York: Wiley, 1968, pt. I.

[12] S. M. Kay, Fundamentals of Statistical Signal Processing. Englewood Cliffs, NJ: Prentice-Hall, 1993.

[13] T. M. Cover and J. A. Thomas, Elements of Information Theory. New York: Wiley, 1991.

[14] J. G. Proakis, Digital Communications. New York, NY: McGraw-Hill, 1995.

[15] C. D. Meyer, Matrix Analysis and Applied Linear Algebra. Philadelphia, PA: SIAM, 2000

[16] M. V. Clark, L. J. Greenstein, W. K. Kennedy, and M. Shafi, "MMSE diversity combining for wideband digital cellular radio," IEEE Trans. Commun., vol. 40, pp. 1128-1135, June 1992.

[17] S. Haykin, Adaptive Filter Theory. Englewood Cliffs, NJ: PrenticeHall, 1991.

[18] S. J. Grant and J. K. Cavers, "Further analytical results on the joint detection of cochannel signals using diversity arrays," IEEE Trans. Commun., vol. 48, pp. 1788-1792, Nov. 2000.

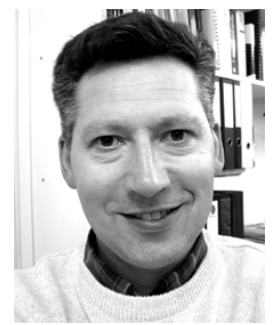

Gerard J. M. Janssen (M'93) received the M.Sc.E.E. degree from Eindhoven University of Technology, Eindhoven, The Netherlands, in 1986 and the Ph.D. degree from Delft University of Technology, Delft, The Netherlands, in 1998.

In 1986, he joined the Physics and Electronics Laboratory, Dutch Organization of Applied Scientific Research (TNO-FEL) The Hague, The Netherlands, where he was involved in radar cross-section modeling, radio direction finding, interference cancellation, and wide-band propagation measurements. He currently is an Associate Professor in the Wireless and Mobile Communications Group, Delft University of Technology. His research interests include wireless communication, especially narrow-band multiuser detection, digital modulation techniques, channel modeling, diversity techniques, and ultrawide-band communications.

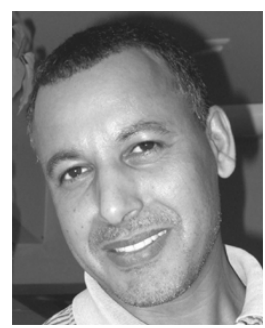

S. Ben Slimane (M'93) received the B.Sc. degree in electrical engineering from the University of Quebec, Trois-Rivieres, PQ, Canada, in 1985 and the M.Sc. and Ph.D. degrees from Concordia University, Montreal, PQ, in 1988 and 1993, respectively.

From 1993 to 1995, he was a Research Associate and part-time Instructor with Concordia University. $\mathrm{He}$ currently is an Associate Professor with the Department of Signals, Sensors, and Systems, Royal Institute of Technlogy (KTH), Stockholm, Sweden. His research interest is in the area of wireless communications with a special emphasis on digital communication techniques for fading channels, error control coding, spread-spectrum communications, and multicarrier transmission techniques. 